\title{
Large animals as models of atrial fibrillation
}

\author{
Piotr Frydrychowski ${ }^{1, B, D}$, Marcin Michałek ${ }^{1, B, D}$, Agnieszka Sławuta ${ }^{2, C-F}$, Agnieszka Noszzzyk-Nowak ${ }^{1, A-F}$ \\ ${ }^{1}$ Department of Internal Medicine and Clinic of Diseases of Horses, Dogs and Cats, Faculty of Veterinary Medicine, Wrocław University of Environmental and Life Sciences, Poland \\ 2 Department of Internal and Occupational Diseases, Hypertension and Clinical Oncology, Wroclaw Medical University, Poland \\ A - research concept and design; $\mathrm{B}$ - collection and/or assembly of data; $\mathrm{C}$ - data analysis and interpretation; \\ $D$ - writing the article; $E$ - critical revision of the article; $F$ - final approval of the article
}

\section{Address for correspondence}

Agnieszka Sławuta

E-mail: aslawuta@tlen.pl

Funding sources

None declared

\section{Conflict of interest}

None declared

Received on September 12, 2019

Reviewed on January 4, 2020

Accepted on May 6, 2020

Published online on June 30, 2020

\begin{abstract}
In clinical practice, atrial fibrillation (AF) is the most common cardiac arrhythmia in humans and it may lead to numerous complications, including central nervous system embolism. The electrical activity of the heart in AF is rapid and chaotic, while the atrioventricular conduction leads to irregular ventricular contraction. Consequently, the stroke volume is reduced, which may lead to symptoms of heart failure. Heart failure is one of the causes of AF as well. Numerous in vivo and in vitro models are used to study the pathophysiology of AF. Animal models play a key role in understanding the mechanisms of arrhythmias as well as in developing treatment regimens. The models of AF include large animals (goats, sheep, pigs, dogs) as well as small laboratory animals. This study reviews the large animal models of $A F$, which enhance our understanding of numerous mechanisms responsible for the development of AF, but we must be aware that the pathomechanism of AF in humans is complex and is affected by numerous factors, including environmental and congenital ones.
\end{abstract}

Key words: arrhythmia, atrial fibrillation, animal models

Cite as

Frydrychowski P, Michałek M, Sławuta A, Noszczyk-Nowak A.

Large animals as models of atrial fibrillation. Adv Clin

Exp Med. 2020;29(6):757-767. doi:10.17219/acem/122130

DOI

10.17219/acem/122130

\section{Copyright}

Copyright by Author(s)

This is an article distributed under the terms of the

Creative Commons Attribution 3.0 Unported (CC BY 3.0)

(https://creativecommons.org/licenses/by/3.0/) 


\section{Introduction}

Atrial fibrillation (AF) is the most frequently diagnosed arrhythmia in humans. ${ }^{1,2}$ It may accompany organic heart disease or occur as a solitary disorder (so-called lone AF), and its nature is multifaceted.

Numerous experimental in vitro and in vivo animal models were developed to understand the pathophysiology of AF and its effects on the organism. However, many questions regarding the mechanisms of AF remain unanswered. Studies with animal models are particularly useful, as they combine data from tissue and cell samples with those from clinical examinations and responses to treatment. Regardless of the type of AF animal model, it will always differ to some extent to AF in humans.

Animal models of AF include large animals (goats, sheep, pigs, dogs) as well as small laboratory animals. ${ }^{3}$ The use of mice, rats or rabbits may be highly advantageous, as these species are easily bred and their maintenance cost is low. In addition, study trials can be frequently repeated. Obtaining genetically modified mice, such as the $\alpha \mathrm{MyHC}$-FKBP12 transgenic mouse that spontaneously develops AF, has enabled their use in electrophysiological studies of AF. However, the use of laboratory animals has many limitations. Due to their small size, all the study procedures are difficult to perform and require the use of specialised, often customized and expensive equipment. Their small size also limits the "study field" of the atria and ventricles. Moreover, the size and availability of the tissue samples (such as heart muscle samples) for further analysis is limited. Although the use of transgenic animals has many benefits, it is very expensive. The lifespan of laboratory animals is relatively short. Hence, some changes observed in humans in the course of AF may never be observed in laboratory animals. This may hinder the long-term assessment of AF in transgenic species. In addition, their physiological parameters such as body temperature, respiratory and heart rate are higher than in humans, which makes it difficult to assess compensatory mechanisms and relate the obtained results to humans. For example, the classification of persistent AF in mice ranges from $1 \mathrm{~min}^{4}$ or $3 \mathrm{~min},{ }^{5}$ while the same condition is recognised in humans with AF persisting longer than 7 days.

The use of large animal (goats, sheep, pigs, and dogs) models may replace certain laboratory animal models. Surgical procedures are easier to perform on large animals. The body mass as well as the heart mass of pigs are similar to those in humans, so more study samples can be obtained. Large animal models also have limitations, such as higher maintenance costs or longer study periods. However, the abovementioned advantages seem to outweigh the limitations, suggesting that large animals are a good model of human AF.

\section{Goats}

Goats were the first animals to be used in studies on AF. In 1995, Wijffels et al. developed a goat model of AF to assess the electrophysiological changes inflicted by longterm AF. ${ }^{6}$ Atrial fibrillation was triggered by pacing the atrium using one-second impulses. After conversion to a sinus rhythm, the pacemaker pulse generator sent impulses to repeat the arrhythmia. This method gave a long-term model of persistent AF, which made it possible to confirm that rapid AF leads to atrial remodeling, affecting the refractory period of the myocardium. Disorders of the refractory period increase the atrial susceptibility to stimuli that induce and maintain AF, meaning that, de facto, AF may beget AF. ${ }^{6}$ Both electrical and structural atrial remodeling were assessed often as a cause of AF. By inflicting a complete atrioventricular block in goats, Neuberger et al. confirmed the hypothesis that atrial dilatation elongates episodes of AF by local conduction delays. ${ }^{7}$ In addition, the atrioventricular (AV) block was used to develop a model that allowed associating atrial contractility disorders with atrial enlargement caused by a slow ventricular rhythm, which develops secondary to AV blocks and atrial remodeling associated with AF. ${ }^{8}$ Van der Velden et al. investigated persistent AF and proved that the reconstruction of the $\mathrm{C} \times 40$ gap junctions may affect the pathogenesis of persistent AF. ${ }^{9}$ In that study, AF was induced using two-second $50 \mathrm{~Hz}$ impulses and maintained from 1 to 16 weeks. ${ }^{9}$ Ausma et al. focused on the influence of remodeling on AF using a goat model. ${ }^{10-12}$ They studied the mutual relations between persistent AF and myocardial structural remodeling. Atrial fibrillation was induced using $50 \mathrm{~Hz}$ impulses. Their studies revealed that persistent AF leads to structural changes in atrial myocytes, which may explain the worse myocardial contractility following cardioversion. ${ }^{10}$ In addition, it was found that goats with AF suffer from progressive cellular structural remodeling in the atrial myocardium, ${ }^{11}$ which is a very slow and reversible process, while remodeling of the junction gaps is completely reversible. ${ }^{12}$ Atrial structural and electrical changes following an induced AV block were also studied by Neuberger et al. in goats. ${ }^{13}$ After applying burst pacing to trigger AF for $48 \mathrm{~h}$, the degree of atrial remodeling was measured. Then, after complete reversal of electrical remodeling, AV block was obtained and the procedure of burst pacing was repeated. Following their measurements, the authors found that electrical remodeling secondary to AF was present in dilated and non-dilated atria. ${ }^{13}$ Remes et al. performed an interesting study with a vascular shunt by connecting the aorta and the left atrium. ${ }^{14}$ The aim of that study was to assess the effect of left atrial overload on the occurrence and maintenance of AF. The results indicated that long-term AF can be a result of chronic atrium overload and occurs 
in most animals without a decrease in the atrial effective refractive period (AERP).

Goats seem to be a good model of atrial remodeling in the course of AF. The mortality rate of the animals in the reported studies was low, and AF was induced relatively easily and in a repeatable manner. This model seems particularly useful in studies requiring long-term persistent AF as it can be maintained for periods of up to 20 weeks, and it does not require specialist equipment or additional procedures. Many of the mechanisms described in the studies on goats are similar to AF in humans. Undeniably, this is beneficial as in the study of the pathomechanism and treatment of AF in humans. The limitations of this model include a lower respiratory rate in goats and a maximal physiological heart rate lying within the lower reference range for humans. In addition, goats require species-specific anesthesia. Due to the fact that ruminants have a unique anatomy and physiology, their anesthesia carries a risk of complications.

\section{Sheep}

Sheep have also been used in cardiac studies, particularly those concerning atrial remodeling. The remodeling was mainly obtained by rapid atrial pacing, sometimes coupled with experimentally induced hypertension or heart failure.

Rapid atrial pacing for AF induction was used by Anné et al. ${ }^{15}$ In addition, Hiss bundle ablation was performed in some of the animals, followed by ventricular pacing at a frequency of $80 \mathrm{bpm}$ and with the administration of quinapril and losartan or a placebo. The results were obtained after a 15-week observation period. The researchers found that there was sufficient atrial electrical remodeling to induce AF. However, structural changes are essential for the occurrence of permanent AF.

Atrial pacing to induce AF was also used in another model, where the structural and functional effects of chronic left atrial dilatation enabled an anastomosis between the aorta and the pulmonary artery. ${ }^{16}$ That procedure increased the blood flow in the pulmonary vein leading to a volume overload. The recording and pacing was carried out using quadripolar electrode placed in the right atrium. The study led to the conclusion that even moderate atrial volume overload may cause significant changes in the electrophysiological properties of the atrial muscle. These changes resemble those observed during AF and are likely to increase the susceptibility to AF. In human medicine, these findings may constitute an early clinical intervention aiming at normalizing hemodynamic parameters associated with atrial overload, which may occur in mitral valve disease. ${ }^{16}$

A modification of the model of atrial remodeling caused by rapid atrial pacing was proposed by Kistler, who studied electrical and structural changes in the atria of sheep with elevated blood pressure. ${ }^{17}$ Hypertension is one of the most common factors involved in the pathogenesis of AF. The authors administered dexamethasone to pregnant sheep on the $27^{\text {th }}$ day of gestation. After obtaining cardiac access, electrodes were sewn into Bachman's bundle and into the appendages and free walls of both atria. As mentioned previously, AF was induced by rapid atrial pacing. A prolongation of AF and an atrial conduction delay were observed in animals with elevated blood pressure compared with the control group. Due to the fact that changes in the structure of the atria, such as atrial hypertrophy and histological remodeling, were also observed, the authors concluded that slow conduction and an increased inductance of AF were associated with structural changes in atria. ${ }^{17}$ Sheep were also used to create a model of heart failure. ${ }^{18}$ That was done by rapid ventricular pacing, which caused dilated cardiomyopathy and dilatation of the left atrium. In all the animals, epicardial electrodes were sewn into the free wall of the left atrium and near right ventricular apex and in some animals into the free wall of the right atrium additionally. The leads were connected to a pacemaker eliciting rapid ventricular pacing (190 beats/min). In the course of the study, 42-day long atrial pacing was performed. A significantly higher atrial susceptibility to AF, a significantly larger LA and prolongation of the atrial effective refractory period were observed. The speed of left atrial enlargement was directly proportional to the increase in AF susceptibility. ${ }^{18}$

Mandapati et al. proposed another ovine model of heart disease using isolated hearts. ${ }^{19}$ Atrial fibrillation was induced by electrodes placed in the left and right atrium. The study enabled exact mapping of the areas of periodic activity during AF. In that model AF was most likely maintained via stable micro re-entrant sources. ${ }^{19}$

Despite some limitations of the sheep model, which are similar in goats, such as a lower respiratory rate, the risk of prion infections or some limitations during echocardiography caused by different anatomical topography of the digestive tract, the sheep is considered a good model for research on AF. In addition, a model of hypertension and a model of heart failure, which are often factors influencing AF, were created using this species. Sheep anesthesia, similarly to that in goats, requires special attention in order to minimize perioperative mortality.

\section{Pigs}

The use of pigs as a model of AF seems promising, as pigs have a similar body mass, heart size, metabolism, and heart rate as humans. These features allow direct translation of the results obtained in these studies into human medicine. As in the abovedescribed sheep and goat models, the developed swine models used rapid atrial pacing and some required non-standard surgical procedures.

Lee et al. aimed to develop a repeatable and simple swine model of maintaining AF, which would not require 
chronic intervention within the atria. ${ }^{20}$ Therefore, they accessed the heart by sternotomy and AF was induced using 3 maneuvers. The $1^{\text {st }}$ maneuver was manual. If that had not induced AF, a pacing lead placed in the upper part of the right atrium was used for rapid atrial pacing, including burst pace. The $3^{\text {rd }}$ maneuver was the administration of neostigmine, leading to an increased vagal tone with shortening of the atrial refractory period. Following neostigmine administration, the previous 2 maneuvers were repeated. If AF lasted longer than $1 \mathrm{~min}$, it was considered continuous. In 14 of the 21 pigs with AF, AF was maintained without neostigmine. This produced a model of acute AF, which can be easily remodelled, as gives reliable results and does not require earlier preparatory procedures. It may be used in studies on ablation procedures and assessing new technologies in the treatment and prevention of AF. ${ }^{20}$ Atrial remodeling caused by rapid atrial pacing was also used to compare the rate of changes in animals with hypertension and normotension. ${ }^{21}$ The six-hour rapid pacing at a frequency of $600 \mathrm{bpm}$ was performed by a pacemaker in the free wall of the right atrium. Digoxin was administered in order to slow down the AV conduction. One group of animals received deoxycorticosterone acetate (DOCA) subcutaneous implants and a salt-rich (4\%) diet for induction of hypertension. Rapid atrial pacing was commenced 2 weeks after the DOCA implantation. The use of this protocol made AF last longer than $60 \mathrm{~min}$. Noticeable dilatation of the left atrium without heart insufficiency was observed 2 weeks after the pacing. The authors also found that AF in this model accelerated the structural remodeling of the atria and stabilised $A F,{ }^{21}$ which confirms the negative effect of hypertension in patients with simultaneous AF.

Linz performed an interesting study using a swine model in order to confirm the hypothesis that low-level baroreceptor stimulation (LL-BRS) may reduce the inducibility of AF in obstructive sleep apnoea (OSA). ${ }^{22}$ The animals in that

Table 1. Individual animal models, taking into account the type of induced AF

\begin{tabular}{|c|c|c|}
\hline Model & Types of induction & Species \\
\hline $\mathrm{AF}$ in an isolated heart & atrial pacing & sheep $^{19}$ \\
\hline \multirow[t]{2}{*}{ Acute AF } & $\begin{array}{c}\text { neostygmine administration, } \\
\text { mechanical cardiac } \\
\text { stimulation, rapid atrial } \\
\text { pacing }\end{array}$ & $\operatorname{pig}^{20}$ \\
\hline & rapid atrial pacing & pig $^{24}$ \\
\hline Long-term AF & long-term atrial pacing & $\begin{array}{c}\text { goat, }^{6} \\
\text { sheep }^{18}\end{array}$ \\
\hline Persistent AF & remodeling of gap junctions & goat $^{9}$ \\
\hline Persistent AF & $\begin{array}{l}\text { rapid atrial pacing, } \\
\text { phenylephrine }\end{array}$ & $\operatorname{dog}^{31}$ \\
\hline $\begin{array}{l}\text { AF resistant } \\
\text { to wernakalant }\end{array}$ & rapid atrial pacing & $\operatorname{pig}^{23}$ \\
\hline $\begin{array}{l}\text { AF in obstructive sleep } \\
\text { apnea }\end{array}$ & $\begin{array}{l}\text { negative tracheal pressures } \\
\text { (ntp), atrial pacing }\end{array}$ & $\operatorname{pig}^{22}$ \\
\hline
\end{tabular}

Table 2. Individual animal models in which induced AF was associated with the structure of the atria and its changes

\begin{tabular}{|c|c|c|}
\hline Model & Types of induction & Species \\
\hline \multirow{5}{*}{ Structural atrial remodeling } & AV block & goat $^{8,13}$ \\
\hline & atrial overload & goat, $^{14}$ sheep $^{16}$ \\
\hline & hypertension & sheep, ${ }^{17}$ pig $^{21}$ \\
\hline & chronic heart failure & sheep, ${ }^{18} \operatorname{dog}^{34,35}$ \\
\hline & rapid atrial pacing & $\begin{array}{c}\text { sheep, }{ }^{15,17} \text { pig, }_{1}{ }^{11} \\
\operatorname{dog}^{25,26,34}\end{array}$ \\
\hline Acute atrial damage & acute ischemia & $\operatorname{dog}^{32,33}$ \\
\hline $\begin{array}{l}\text { Foci sensitive to catecho- } \\
\text { lamines in the course of AF }\end{array}$ & rapid atrial pacing & $\operatorname{dog}^{28}$ \\
\hline $\begin{array}{l}\text { Focal activity in pulmonary } \\
\text { veins and atria in persistent } \\
\text { AF }\end{array}$ & rapid atrial pacing & $\operatorname{dog}^{30}$ \\
\hline $\begin{array}{l}\text { Involvement of pulmonary } \\
\text { veins and the vein } \\
\text { of Marshall in AF }\end{array}$ & $\begin{array}{l}\text { atrial pacing, } \\
\text { chronic heart failure }\end{array}$ & $\operatorname{dog}^{36}$ \\
\hline
\end{tabular}

study underwent a tracheotomy to obtain negative tracheal pressure (NTP). Following AF induction via atrial pacing, carotid baroreceptor stimulation was performed. The obtained results confirmed that low-level baroreceptor stimulation inhibited the NTP-induced shortening of the atrial refractory period and induction of AF. These results confirm the use of LL-BRS as a novel therapeutic method in the treatment of AF in obstructive sleep apnoea. ${ }^{22}$ Pigs were also used to study new therapies of drug-resistant AF. Diness et al. created a model of AF to verify whether the SK channel inhibitor, known as AP14145, can effectively terminate AF resistant to vernakalant. ${ }^{23}$ To begin with, they received seven-day pacing of the right atrium at a frequency of $420 \mathrm{bpm}$ in order to induce sustained AF. Then, they placed electrodes into both the left atrial appendage and the left ventricle for the purpose of recording and pacing. After persistent AF was confirmed, the pigs received vernakalant as pharmacological cardioversion. If that had proven successful, the animals received repeated seven-day pacing, followed by vernakalant administration. If AF did not convert to sinus rhythm (SR), the animals received AP14145 intravenously.

Rapid atrial pacing aimed at inducing AF was also used by Jones et al. They carried out a study on pigs using $\mathrm{CARTO}^{\circledR}$ mapping to identify anatomical causes of conduction disturbances triggered by poorly overlapping ablation lines, leading to incomplete pulmonary vein isolation. ${ }^{24}$ Atrial fibrillation was achieved by rapid atrial pacing. The pulmonary veins were isolated using cryoisolation. The authors found that their results were similar to those in patients with persistent or paroxysmal AF, which indicates that the swine model is a valuable experimental model of AF. ${ }^{24}$

In pigs, like in other animals, rapid ventricular pacing was used to cause congestive heart failure. However, unlike the aforementioned study on the ovine model, ${ }^{18}$ no higher atrial susceptibility to AF or the occurrence of the arrhythmia of was observed. ${ }^{25}$ 
The swine model of AF proved easily inducible and useful in studies on novel AF therapies. Rapid atrial pacing, which induced AF, is a simple and repeatable procedure in pigs.

\section{Dogs}

Dogs have been frequently used to develop models to better understand AF. The simplicity of surgical procedures in this species is an unquestionable advantage, allowing various studies to use dogs of different weights and sizes. An important factor in favor of canine models is the possibility of studying AF in animals with heart failure or atrial ischemia. In addition, due to the size of dogs, certain devices and equipment are suitable for pediatric electrotherapy. At the same time, dogs have a higher natural heart rate than humans, and this is one of the limitations of the canine model. Still, dogs have turned out to be particularly useful in the study of the parameters and atrial electrophysiological changes that lead to AF or contribute to its maintenance. In addition, dogs were used to determine the mechanism and effect of certain drugs on AF, which increased the putative pool of antiarrhythmic drugs.

The effect of drugs on atrial remodeling and electrophysiology parameters was reported by Shiroshita-Takeshita et al. ${ }^{26,27}$ The authors assessed the effect and mechanisms of simvastatin, vitamin $C$ or vitamin $C$ and $E$ on a model of AF induced by atrial tachypacing (ATP). An AV block was induced to control the ventricular rhythm. ${ }^{26}$ In another study, Shiroshita-Takeshita et al. assessed the effect of ibuprofen on the tachycardia-remodeling in dogs. ${ }^{27}$ The authors used the same model of ATP as in the previously mentioned study but the dogs were administered ibuprofen, cyclosporine A or prednisone. ${ }^{27}$ Rapid atrial pacing was also used by Gaspo et al. ${ }^{28}$ In their model, the lead in the right atrium was connected to a pacemaker placed subcutaneously in the neck area. The pacing frequency was set at $400 \mathrm{bpm}$ and lasted 1, 7 and 42 days, depending on the group. Atrial fibrillation was induced using $2 \mathrm{~ms}$ impulses at $10 \mathrm{~Hz}$. If the AF exceeded $45 \mathrm{~min}$, it was considered long-term and was cardioverted. The study indicated that chronic atrial tachycardia not only reduces AERP (atrial effective refractory period), the conduction time and the wavelength, but also increases the capacity of atrial extrasystoles to induce and extend the duration of AF. The study was valuable, as it enabled an assessment of electrophysiological changes crucial for persistent AF. ${ }^{28}$ Doshi et al. used a canine model to localize catecholaminesensitive foci in the left atrium and then assessed their activity during AF induced via rapid atrial pacing. That study required in vivo and in vitro assessment. First, some dogs underwent a cardiectomy in order to collect samples for the in vitro and electrophysiological studies, including an infusion of isoproterenol into the coronary artery. In the remaining dogs, a pacing lead in the right atrium was connected to a subcutaneous pacemaker. The animals were monitored periodically for long-term AF, which was recorded in dogs 10,11 and 41 weeks after lead placement. The authors found that the isoproterenol infusion may induce activity in the ligament of Marshall, and that this activity is higher in dogs with AF induced by tachypacing. It was also observed that rapid activity during the isoproterenol infusion may induce AF in vitro in the left atrium of dogs with long-term AF. This phenomenon is not observed in healthy atria. The results led to the conclusion that heightened sensitivity to isoproterenol following pacing may contribute to the development of AF in the described model. ${ }^{29}$ The same method of AF induction was used to test the hypothesis that long-term stimulated AF is characterized by the presence of chaotic waves as well

\begin{tabular}{|c|c|}
\hline $\begin{array}{c}\text { RIGHT ATRIUM } \\
\text { Rapid atrial pacing }\end{array}$ & $\begin{array}{c}\text { LEFT ATRIUM } \\
\text { Rapid atrial pacing } \\
\text { Vascular shunt (aorta and LA; aorta and } \\
\text { pulmonary artery) } \\
\text { Isoproterenol }\end{array}$ \\
Complete atrioventricular block - atrial dilatation elongating episodes of AF \\
Atrial contractility disorders associated with atrial enlargement \\
Persistent AF leading to structural changes \\
Neostigmine \\
Manual maneuver \\
Low level baroreceptor stimulation in obstructive sleep apnea \\
Atrial ischemia (ligating the branch of the right coronary artery) \\
Arrhythmogenic atrial remodeling (angiotensin II)
\end{tabular}

Fig. 1. The mechanisms of $A F$ in animal models 

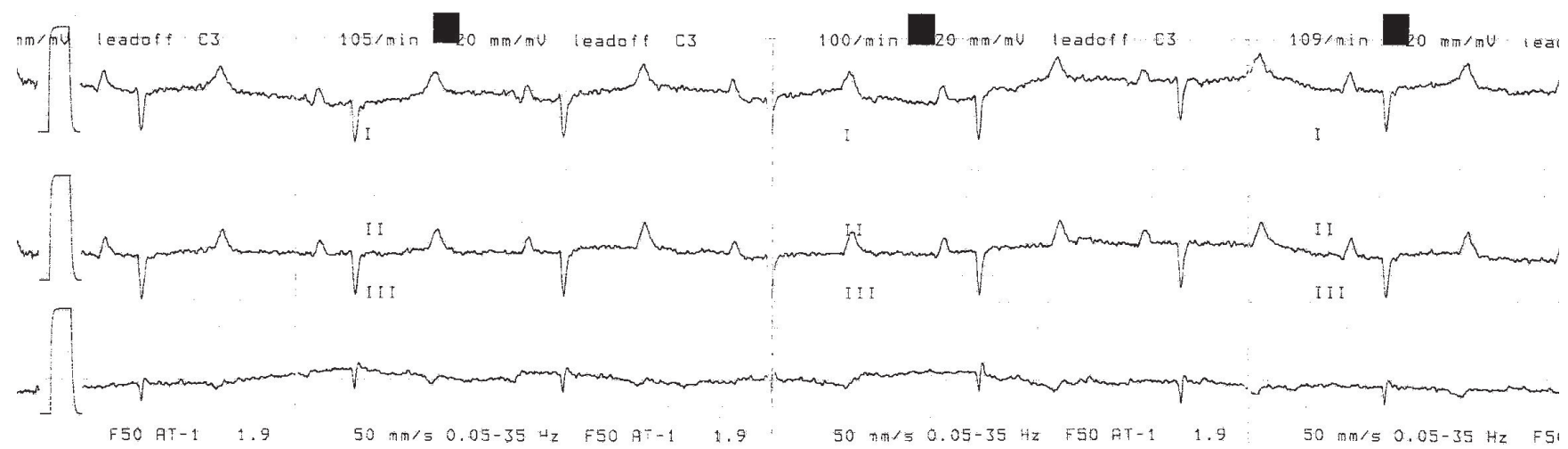

Fig. 2. Sinus rhythm in sheep

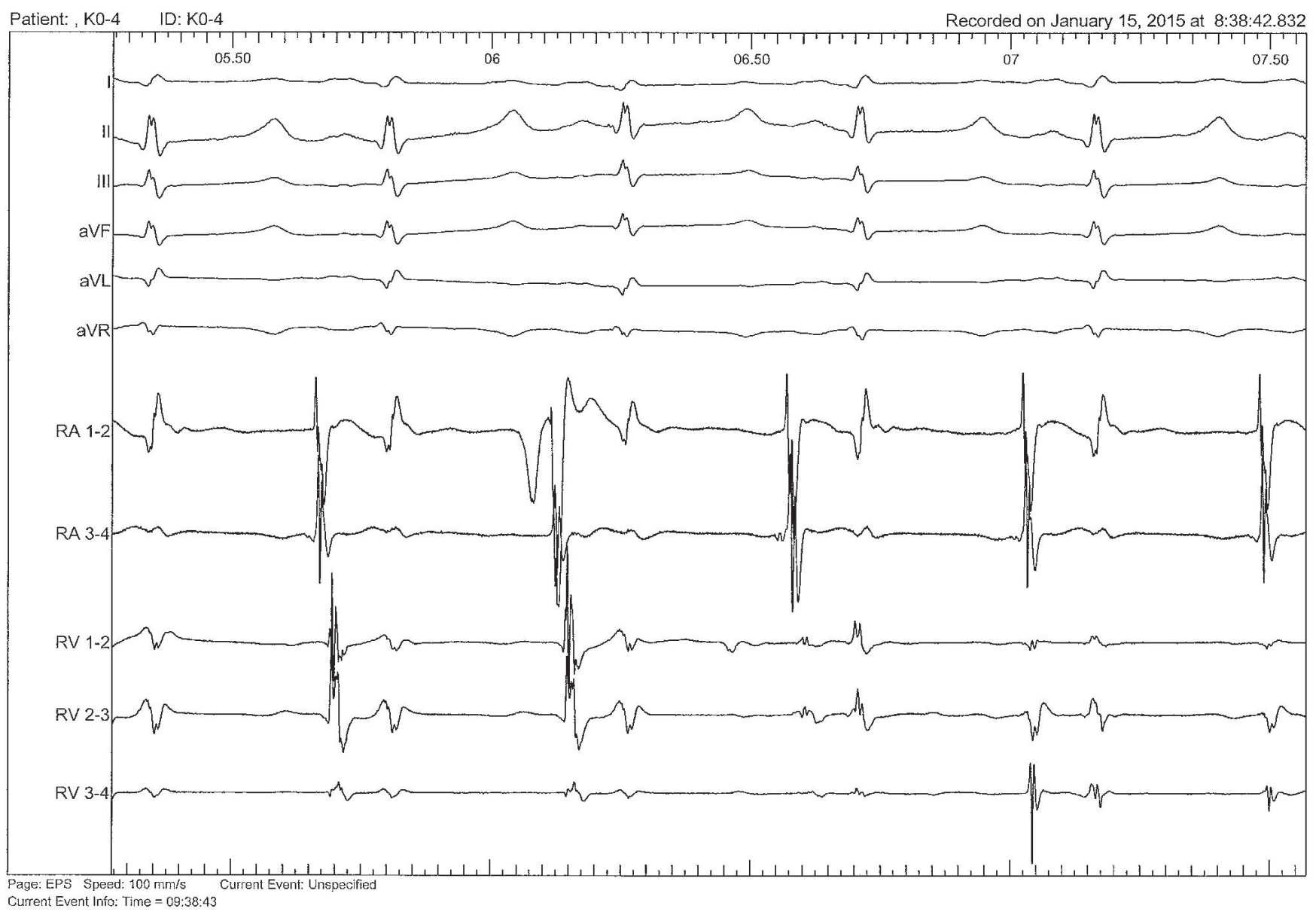

Fig. 3. Sinus rhythm in pig

as organized re-entry waves. ${ }^{30} \mathrm{~A}$ bipolar electrode connected with a pacemaker was inserted into the right atrium. After confirming AF, the animals underwent mapping. The study confirmed the presence of chaotic disorganized and organized re-entry waves during persistent AF. In addition, the authors concluded that the ligament of Marshall and the pulmonary veins are the sources of rapid activation in the course of long-term AF induced by chronic stimulation. ${ }^{30}$ Electrophysiological studies using a model based on rapid pacing were also performed by Zhou et al. to determine the patterns of activity in the pulmonary veins and atria during $\mathrm{AF} .{ }^{31}$ Intermittent rapid pacing was performed using a neurostimulator connected to a screw-in bipolar lead placed in the left atrium appendage in order to induce continuous AF. The pulse generator was programmed to burst with pacing interval of $50 \mathrm{~ms}$ for $5 \mathrm{~s}$, followed by a two-second period without pacing. The arrhythmia was considered continuous if it persisted for $>48 \mathrm{~h}$ after the pacemaker was turned off. This study found that rapid focal activations were present in the pulmonary veins following persistent AF caused by left atrium pacing. Such activation pattern is not present in pulmonary veins during 


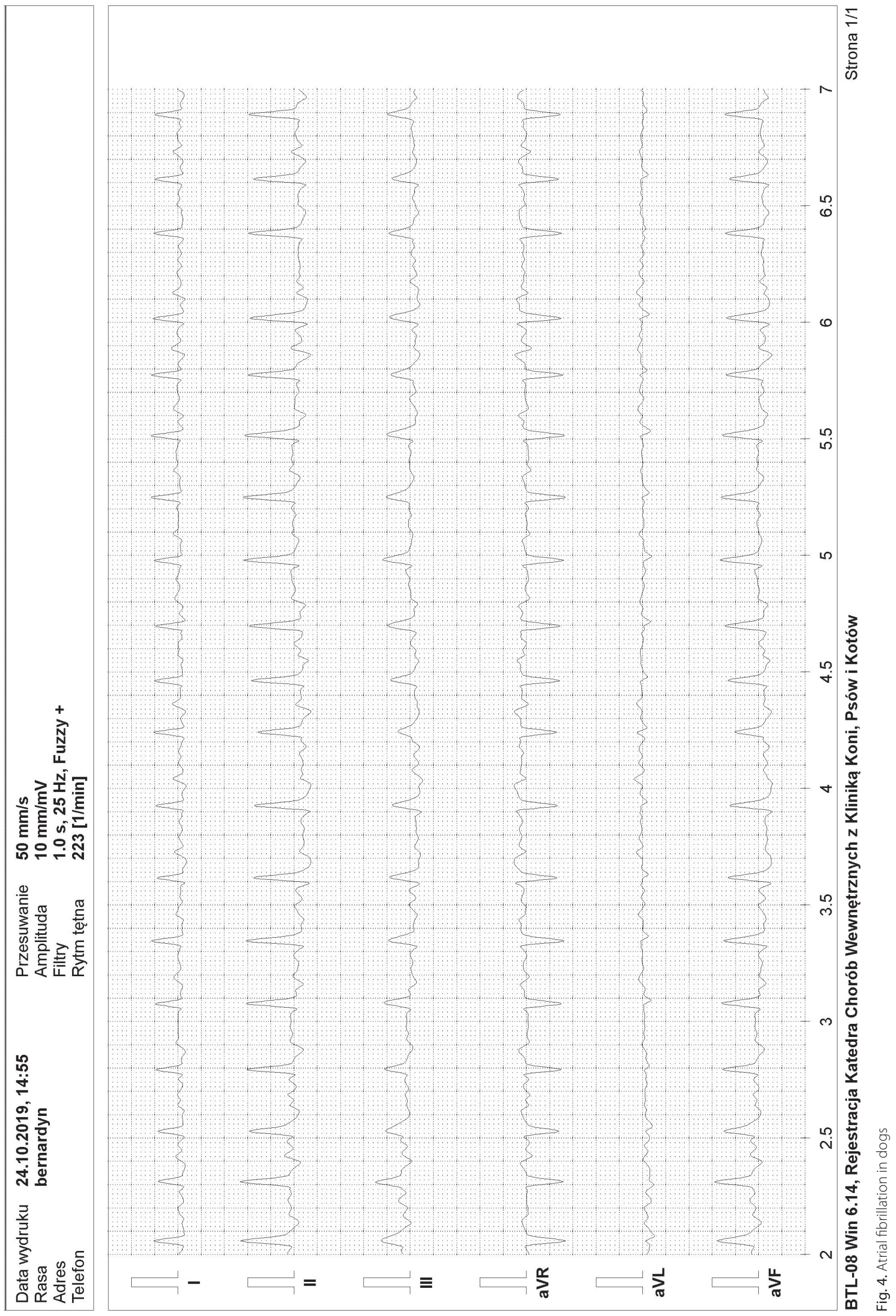




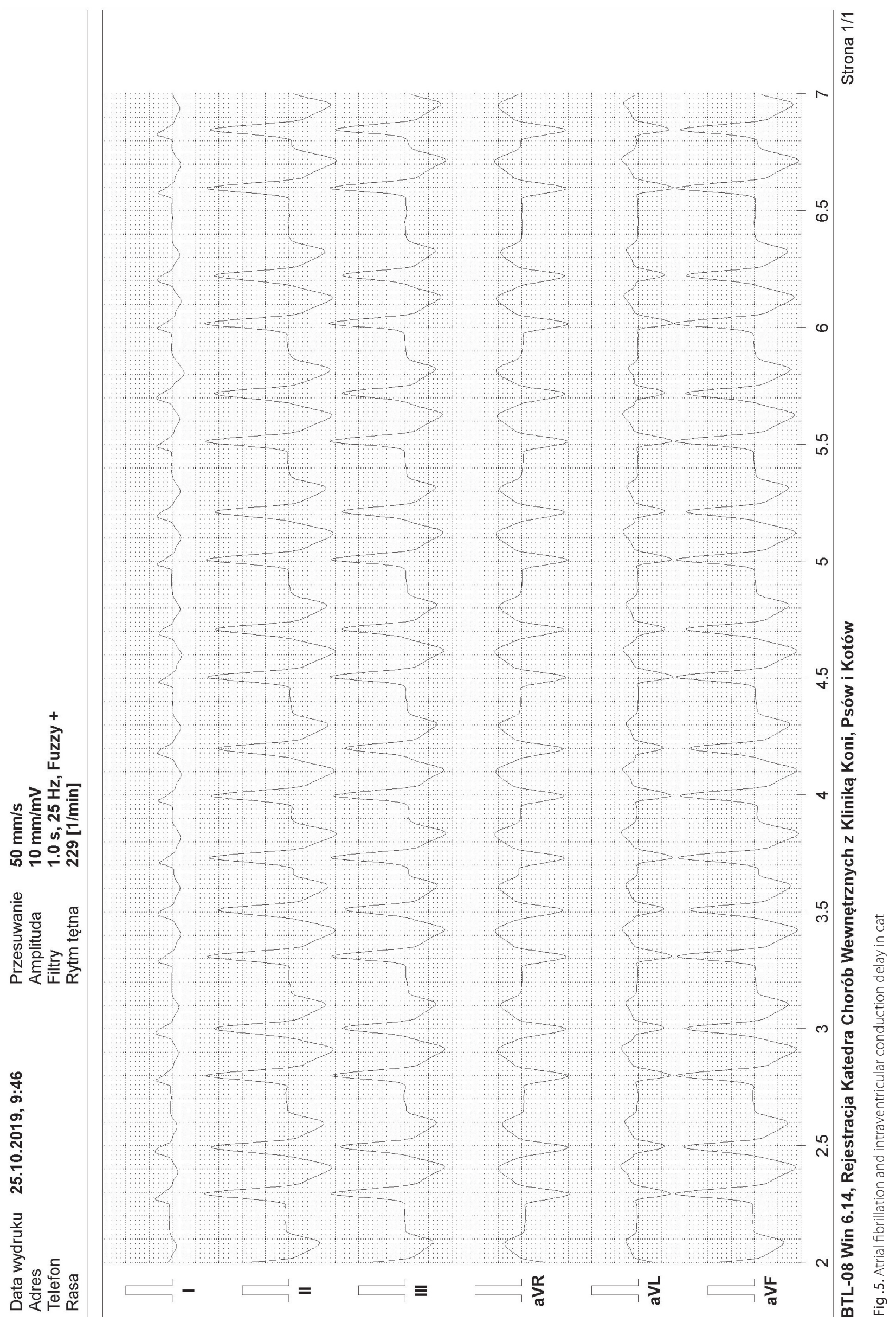


non-persistent AF. The results of that study confirmed the hypothesis that the pulmonary veins and ligament of Marshall are sources of rapid focal activations in a canine model of induced AF. ${ }^{31}$

The maintenance of AF in these studies required rapid pacing for extended periods of time. Therefore, studies on the AF model were carried out in such a way that the AF could be maintained for a sufficiently long time to perform all the necessary tests. Kijtaworn rat et al. used atrial stimulation with $2 \mathrm{~ms}$ waves at a frequency of $40 \mathrm{~Hz}{ }^{32}$ Some dogs received carbachol and phenylephrine in addition to the stimulation, which was ended after $20 \mathrm{~min}$. The heart rate was then assessed in order to identify and confirm the induction of AF. In half of the tested dogs, the administration of phenylephrine causing an increase in arterial pressure led to the maintenance of AF for a period longer than $40 \mathrm{~min}^{32}$

A canine model of ischemia was also developed by Sinno et $a .^{33}$ In the study, atrial ischemia was achieved by ligating the branch of the right coronary artery. Atrial fibrillation was induced using $10 \mathrm{~Hz}$ impulse from leads placed in the left and right atrium appendage. The authors concluded that atrial ischemia maintains AF through slowing of conduction. The results of that study may be implemented in patients with coronary disease. ${ }^{33}$ The results of that study and the described model were used to assess the effect of antiarrhythmic drugs on the electrophysiological consequences of acute atrial ischemia. ${ }^{34}$ The authors also compared the effect of the drugs on ischemic and vagal AF. The ischemia model was obtained analogously to that in the study by Sinno et al. ${ }^{33}$ The dogs were divided into groups including animals without treatment that underwent pacing, paced dogs that received diltiazem, nadolol, flecainide or dofetilide, and dogs with vagal AF. The study showed that that some drugs effectively prevent changes in atrial conduction and AF in acute atrial ischemia but the effects of these drugs on vagal AF were quite different. The obtained results may be of value for studies assessing the variable efficacy of antiarrhythmic drugs in the treatment of AF. ${ }^{34}$

The assessment of changes leading to cardiac remodeling and the development of AF in congestive heart failure $(\mathrm{CHF})$ is especially important. As is well known, this arrhythmia may lead to the occurrence and progression of heart failure, although heart failure itself is a known risk factor of AF. Therefore, models based on CHF appear to be extremely helpful in studies on the induction and course of AF. Dogs were also used to create this type of model. In their study, Li et al. described the electrophysiological and structural changes in the atria caused by experimentally induced chronic heart failure. ${ }^{35}$ The authors also wanted to verify whether those changes could facilitate the induction of AF and to compare atrial remodeling in CHF with that caused by rapid atrial pacing. In the dogs, heart failure was induced by rapid ventricular pacing. In another group of dogs, the atria were paced at $400 \mathrm{bpm}$. Atrial fibrillation was induced by applying a maximum of 3 consecutive extrastimuli, followed by atrial pacing with impulses at $10 \mathrm{~Hz}$ from $1 \mathrm{~s}$ to $10 \mathrm{~s}$. Atrial fibrillation was considered persistent if it lasted longer than $30 \mathrm{~min}$ and needed to be discontinued by cardioversion. The study found that chronic heart failure favors persistent AF through a mechanism other than chronic atrial tachycardia. ${ }^{35}$ In another study, Li et al. assessed the involvement of angiotensin II activation and mitogenactivated protein kinases (MAPK) in the induction of AF that promotes structural atrial remodeling. ${ }^{36}$ Canine models were used to assess changes in atrial angiotensin II and MAPK in chronic heart failure caused by ventricular tachypacing (VTP) and the effects of ACE-I (angiotensin converting enzyme inhibitors) on the atrial angiotensin II, MAPK and the development of AF. ${ }^{36}$ Ventricular pacing was carried out according to a methodology described by the same author, ${ }^{35}$ while AF was induced by atrial pacing using $10 \mathrm{~Hz}$ impulses. Atrial fibrillation was considered persistent if it lasted longer than $20 \mathrm{~min}$. The study found that ventricular pacing increases the concentration of atrial angiotensin II and MAPK. Enalapril suppressed those substances and was found to reduce the arrhythmogenic atrial remodeling. ${ }^{36}$ Okuyama et al. used a canine model associated with CHF in order to confirm the hypothesis that proarrhythmic remodeling occurs in the tunica media of the thoracic veins. ${ }^{37}$ Similar conclusions were reached by Wu et al. ${ }^{30}$ The dogs underwent ventricular pacing in order to induce chronic heart failure. Then, the device was switched off and atrial tachyarrhythmia (AT) was induced by leads placed in the right atrium or the left atrial appendage. The study showed that some AF episodes in dogs with $\mathrm{CHF}$ are characterized by the presence of focal activation in the pulmonary veins and the vein of Marshall (oblique vein of the left atrium), suggesting their role in atrial arrhythmias in chronic heart failure. Pulmonary veins may also play a role in the progression of AT to AF. ${ }^{37}$

\section{Cats}

Cats have also been used as animal models in AF studies. The research focused mainly on supraventricular arrhythmias induced with aconitin; however, only a few studies describing experiments with this substance are available in the literature. ${ }^{38-43}$ Ammar and Kudrin were the first to describe the effects of aconitin administration to the sinus node on the heart rhythm. ${ }^{38}$ Gendenshtein et al. induced AF by local administration of $0.05 \%$ aconitin into the right atrium. ${ }^{39}$ Byrne and his team, in turn, used a saturated solution of aconitin, which successfully induced AF in 3 out of 5 tested cats. ${ }^{40}$ Winslow also tested the arrhythmogenic effect of aconitin. He conducted his experiment on anesthetized cats in whom arrhythmia was induced by a local application to the middle part of the left atrial appendage of $0.04 \%$ aconitin nitrate solution. The administration was performed using a cotton swab moistened 
with the aconitin solution in a polyethylene tube. ${ }^{41}$ Winslow created a model in which supraventricular arrhythmias, including AF, can be easily induced by topical administration of aconitin. In addition, this model may be useful in antiarrhythmic drugs research. This model proved to be more effective in inducing AF than those tested in previous experiments. ${ }^{38-40,42-44}$ It is worth mentioning that AF may be developed secondary to left atrial enlargement in the natural course of feline hypertrophic cardiomyopathy.

\section{New perspectives in antiarrhythmic therapy in animal models}

TWIK-related acid-sensitive $\mathrm{K}^{+}$(TASK) channels belong to the two-pore-domain potassium (K2P) channel family. They are relevant for the pathogenesis of many different cardiovascular diseases including AF. TASK-1 channels are promising drug targets for the treatment of AF. The expression and function of those channels is enhanced in atrial cardiomyocytes of patients with chronic AF resulting in action potential duration shortening. The inhibition of TASK-1 in rodents caused prolongation of action potentials. The mechanism previously demonstrated in rodents was successfully elucidated in porcine model by Schmidt et al. ${ }^{45}$ As porcine channel protein is almost identical to human, this model is perfect for the experiments with novel antiarrhythmic target. In recent studies, Schmidt et al. showed that genetic ablation of TASK-1 in porcine model significantly reduced AF burden, which was associated with the reduction of TASK-1 currents and the prolongation of action potential duration. ${ }^{46}$ In search for a perfect animal model for research on the latest antiarrhythmic drugs, Wiedmann et al. studied transgenic mice and concluded that murine, porcine and human TASK-1 channels share many functional similarities. ${ }^{47}$

\section{Conclusions}

The above discussion presents animal models used to assess AF. To date, numerous models based on various mechanisms related to this disorder have been created, although none is perfect. The pathomechanism of AF in humans is complex and is affected by numerous factors, including environmental and congenital ones. Even the most advanced animal model of AF is unable to reflect the complexity and diversity of the etiopathogenesis of this arrhythmia. The use of animals as models of AF is flawed to some extent due to the fact that this type of model imitates chosen AF pathophysiological changes. Species differences affect the development and the course of diseases. However, these models enhance our understanding of numerous mechanisms responsible for the development of AF as well as new therapies to cure this type of arrhythmia. The knowledge and information obtained from the results of the described studies of AF enable a better understanding of the causes and principles of AF, which will hopefully lead to the development of new treatment and preventive methods. All the conclusions drawn from the animal studies of AF need to be analyzed with care, considering differences to the human organism. Studies using mathematical models based on animal models are gaining popularity, as they may enable scientific research without the need to utilize live animals.

\section{ORCID iDs}

Piotr Frydrychowski (D) https://orcid.org/0000-0001-6688-3051 Marcin Michałek (D) https://orcid.org/0000-0002-3762-1201 Agnieszka Sławuta (D) https://orcid.org/0000-0001-5671-9864 Agnieszka Noszczyk-Nowak (D) https://orcid.org/0000-0001-7899-3936

\section{References}

1. Allessie MA, Boyden PA, Camm AJ, et al. Pathophysiology and prevention of atrial fibrillation. Circulation. 2001;103(5):769-777.

2. Noszczyk-Nowak A, Pasławska U, Zyśko D, Gajek J, Nicpoń J, Hebel M. Migotanie przedsionków u psów. Medycyna Wet. 2008;64:686-689.

3. Saunders A, Gordon S, Miller M. Canine atrial fibrillation. Compend Contin Educ Vet. 2009;31(11):E1-9.

4. Ninio DM, Saint DA. Passive pericardial constraint protects against stretch-induced vulnerability to atrial fibrillation in rabbits. Am J Physiol Heart Circ Physiol. 2006;291(5):H2547-2549.

5. Henry BL, Gabris B, Li Q, et al. Relaxin suppresses atrial fibrillation in aged rats by reversing fibrosis and upregulating $\mathrm{Na}^{+}$channels. Heart Rhythm. 2016;13(4):983-991.

6. Wijffels MC, Kirchhof CJ, Dorland R, Allessie MA. Atrial fibrillation begets atrial fibrillation: A study in awake chronically instrumented goats. Circulation. 1995;92(7):1954-1968.

7. Neuberger HR, Schotten U, Verheule S, et al. Development of a substrate of atrial fibrillation during chronic atrioventricular block in the goat. Circulation. 2005;111(1):30-37.

8. Greiser M, Neuberger HR, Harks E, et al. Distinct contractile and molecular differences between two goat models of atrial dysfunction: AVblock-induced atrial dilatation and atrial fibrillation.JMol Cell Cardiol. 2009;46(3):385-394.

9. van der Velden HM, Ausma J, Rook MB, et al. Gap junctional remodeling in relation to stabilization of atrial fibrillation in the goat. Cardiovasc Res. 2000;46(3):476-486.

10. Ausma J, Wijffels M, Thoné F, Wouters L, Allessie M, Borgers M. Structural changes of atrial myocardium due to sustained atrial fibrillation in the goat. Circulation. 1997;96(9):3157-3163.

11. Ausma J, Litjens $\mathrm{N}$, Lenders $\mathrm{MH}$, et al. Time course of atrial fibrillation-induced cellular structural remodeling in atria of the goat. $J \mathrm{Mol}$ Cell Cardiol. 2001;33(12):2083-2094.

12. Ausma J, van der Velden HM, Lenders $\mathrm{MH}$, et al. Reverse structural and gap-junctional remodeling after prolonged atrial fibrillation in the goat. Circulation. 2003;107(15):2051-2058.

13. Neuberger HR, Schotten U, Blaauw $Y$, et al. Chronic atrial dilation, electrical remodeling, and atrial fibrillation in the goat. J Am Coll Cardiol. 2006;47(3):644-653.

14. Remes J, van Brakel TJ, Bolotin G, et al. Persistent atrial fibrillation in a goat model of chronic left atrial overload. J Thorac Cardiovasc Surg. 2008;136(4):1005-1011.

15. Anné $W$, Willems $R$, Holemans $P$, et al. Self-terminating AF depends on electrical remodeling while persistent $A F$ depends on additional structural changes in a rapid atrially paced sheep model. $J$ Mol Cell Cardiol. 2007;43(2):148-158.

16. Deroubaix E, Folliguet T, Rücker-Martin C, et al. Moderate and chronic hemodynamic overload of sheep atria induces reversible cellular electrophysiologic abnormalities and atrial vulnerability. J Am Coll Cardiol. 2004;44(9):1918-1926. 
17. Kistler PM, Sanders P, Dodic M, et al. Atrial electrical and structural abnormalities in an ovine model of chronic blood pressure elevation after prenatal corticosteroid exposure: Implications for development of atrial fibrillation. Eur Heart J. 2006;27(24):3045-3056.

18. Power JM, Beacom GA, Alferness CA, et al. Susceptibility to atrial fibrillation: A study in an ovine model of pacing-induced early heart failure. J Cardiovasc Electrophysiol. 1998;9(4):423-435.

19. Mandapati R, Skanes A, Chen J, Berenfeld O, Jalife J. Stable microreentrant sources as a mechanism of atrial fibrillation in the isolated sheep heart. Circulation. 2000;101(2):194-199.

20. Lee AM, Miller JR, Voeller RK, et al. A simple porcine model of induc ible sustained atrial fibrillation. Innovations (Phila). 2016;11(1):76-78.

21. Schwarzl M, Alogna A, Zweiker D, et al. A porcine model of early atrial fibrillation using a custom-built, radio transmission-controlled pacemaker. J Electrocardiol. 2016;49(2):124-131.

22. Linz D, Hohl M, Khoshkish S, et al. Low-level but not high-level baroreceptor stimulation inhibits atrial fibrillation in a pig model of sleep apnea. J Cardiovasc Electrophysiol. 2016;27(9):1086-1092.

23. Diness JG, Skibsbye L, Simó-Vicens R, et al. Termination of vernakalant-resistant atrial fibrillation by inhibition of small-conductance $\mathrm{Ca}(2+)$-activated $\mathrm{K}(+)$ channels in pigs. Circ Arrhythm Electrophysiol. 2017;10(10):e005125.

24. Jones DL, Guiraudon GM, Skanes AC, Guiraudon CM. Anatomical pitfalls during encircling cryoablation of the left atrium for atrial fibrillation therapy in the pig. JInterv Card Electrophysiol. 2008;21(3):187-193.

25. Paslawska U, Gajek J, Kiczak L, et al. Development of porcine model of chronic tachycardia-induced cardiomyopathy. Int J Cardiol. 2011; 153(1):36-41.

26. Shiroshita-Takeshita A, Schram G, Lavoie J, Nattel S. Effect of simvastatin and antioxidant vitamins on atrial fibrillation promotion by atrial-tachycardia remodeling in dogs. Circulation. 2004;110(16): 2313-2319.

27. Shiroshita-Takeshita A, Brundel BJ, Lavoie J, Nattel S. Prednisone prevents atrial fibrillation promotion by atrial tachycardia remodeling in dogs. Cardiovasc Res. 2006;69(4):865-875.

28. Gaspo R, Bosch RF, Talajic M, Nattel S. Functional mechanisms underlying tachycardia-induced sustained atrial fibrillation in a chronic dog model. Circulation. 1997;96(11):4027-4035.

29. Doshi RN, Wu TJ, Yashima M, et al. Relation between ligament of Marshall and adrenergic atrial tachyarrhythmia. Circulation. 1999;100(8): 876-883.

30. Wu TJ, Ong JJ, Chang CM, et al. Pulmonary veins and ligament of Marshall as sources of rapid activations in a canine model of sustained atrial fibrillation. Circulation. 2001;103(8):1157-1163.

31. Zhou S, Chang CM, Wu TJ, et al. Nonreentrant focal activations in pulmonary veins in canine model of sustained atrial fibrillation. Am J Physiol Heart Circ Physiol. 2002;283(3):H1244-H1252.

32. Kijtawornrat A, Roche BM, Hamlin RL. A canine model of sustained atrial fibrillation induced by rapid atrial pacing and phenylephrine. Comp Med. 2008;58(5):490-493.
33. Sinno H, Derakhchan K, Libersan D, Merhi Y, Leung TK, Nattel S. Atrial ischemia promotes atrial fibrillation in dogs. Circulation. 2003;107(14): 1930-1936.

34. Rivard L, Sinno H, Shiroshita-Takeshita A, Schram G, Leung TK, Nattel $\mathrm{S}$. The pharmacological response of ischemia-related atrial fibrillation in dogs: Evidence for substrate-specific efficacy. Cardiovasc Res. 2007;74(1):104-113.

35. Li D, Fareh S, Leung TK, Nattel S. Promotion of atrial fibrillation by heart failure in dogs: Atrial remodeling of a different sort. Circulation. 1999;100(1):87-95.

36. Li D, Shinagawa K, Pang L, et al. Effects of angiotensin-converting enzyme inhibition on the development of the atrial fibrillation substrate in dogs with ventricular tachypacing-induced congestive heart failure. Circulation. 2001;104(21):2608-2614.

37. Okuyama Y, Miyauchi Y, Park AM, et al. High resolution mapping of the pulmonary vein and the vein of Marshall during induced atrial fibrillation and atrial tachycardia in a canine model of pacing-induced congestive heart failure. J Am Coll Cardiol. 2003;42(2):348-360.

38. Ammar EM, Kudrin AN. Comparative antiarrhythmic activity of beta$\mathrm{N}$-hexamethyleneimino-P-butoxypropiophenone, quinidine and novocaine amide in aconitine auricular fibrillation and flutter in cats. Farmakol Toksikol. 1969;32(4):415-418.

39. Gendenshteĭn El, Kostin IV, Simon IB. Anti-arrhythmic activity of the beta2-adrenoblockader alpheprol. Biull Eksp Biol Med. 1976;81(6): 694-696.

40. Byrne JE, Gomoll AW, McKinney GR. Antiarrhythmic properties of MJ 9067 in acute animal models. J Pharmacol Exp Ther. 1977;200(1):147-154.

41. Winslow E. Hemodynamic and arrhythmogenic effects of aconitine applied to the left atria of anesthetized cats: Effects of amiodarone and atropine. J Cardiovasc Pharmacol. 1981;3(1):87-100.

42. Gendenshteĭn El, Kostin IV. Antiarrhythmic activity of trimecaine in experimental arrhythmia and its effect on the heart conduction system. Farmakol Toksikol. 1976;39(4):426-428.

43. Gendenshteĭn El, Kostin V, Volkova ND. Antiarrhythmic activity of adrenergic blockaders with different mechanisms of action. Kardiologiia. 1977;17(4):116-120.

44. Kaverina NV, Senova ZP, Vikxliàev lul, Ul'iànova OV. Antiarrhythimic properties of ethmozine. Farmakol Toksikol. 1970;33(6):693-697.

45. Schmidt C, Wiedmann F, Langer $C$, et al. Cloning, functional characterization, and remodeling of K2P3.1 (TASK-1) potassium channels in a porcine model of atrial fibrillation and heart failure. Heart Rhythm. 2014;11(10):1798-1805.

46. Schmidt C, Wiedmann F, BeyersdorfC, et al. Genetic ablation of TASK-1 (tandem of $\mathrm{P}$ domains in a weak inward rectifying $\mathrm{K}(+)$ Channel-related acid-sensitive $\mathrm{K}(+)$ Channel-1) (K(2P)3.1) K(+) channels suppresses atrial fibrillation and prevents electrical remodeling. Circ Arrhythm Electrophysiol. 2019;12(9):e007465.

47. Wiedmann F, Schulte JS, Gomes B, et al. Atrial fibrillation and heart failure-associated remodeling of two-pore-domain potassium (K(2P)) channels in murine disease models: Focus on TASK-1. Basic Res Cardiol. 2018;113(4):27. 